\title{
Application of Fiber Bragg Grating Acoustic Emission Sensors in Thin Polymer-Bonded Explosives
}

\author{
Tao Fu ${ }^{1}$, Peng Wei ${ }^{2}, * \mathbb{D}$, Xiaole Han ${ }^{2}$ ) and Qingbo Liu ${ }^{2}$ \\ 1 Institute of Chemical Materials, China Academy of Engineering Physics, Mianyang 621900, China; \\ futao@caep.cn \\ 2 School of Instrumentation and Optoelectronic Engineering, Beihang University, Beijing 100191, China; \\ hanxiaole@buaa.edu.cn (X.H.); liuqingbo@buaa.edu.cn (Q.L.) \\ * Correspondence: weipeng@buaa.edu.cn; Tel.: +86-10-8233-9609
}

Received: 13 October 2018; Accepted: 31 October 2018; Published: 5 November 2018

\begin{abstract}
Fiber Bragg grating (FBG) acoustic emission (AE) sensors have been used in many applications. In this paper, based on an FBG AE sensor, the sensing principle of the interaction between the AE wave and the sensor is introduced. Then, the directionality of the FBG AE sensor on the surface of a thin polymer-bonded explosive (PBX) material is studied. Finally, the time coefficient location method is proposed to correct the AE time detected by the FBG AE sensor, thereby improving the accuracy of location experiments.
\end{abstract}

Keywords: fiber Bragg grating acoustic emission sensor; directionality; time coefficient location method; polymer-bonded explosives

\section{Introduction}

Polymer-bonded explosive (PBX) materials are popular materials for use in weapons because of their advanced high-energy performance. To safely store a PBX in a narrow container, a thin PBX is produced; however, when the environmental temperature changes, cracks can form on this critical material because of its low thermal conductivity. As a result, the risk of explosion of the thin PBX material will increase. Therefore, a stable and reliable nondestructive testing method is urgently needed to monitor the health of thin PBX materials.

Acoustic emission (AE) detection is a nondestructive testing method. Since the initial development of $\mathrm{AE}$ theory in the 1950s, AE detection has been successfully used in the aerospace, machinery manufacture, petrochemical industries and other fields [1]. Currently, a piezoelectric (PZT) sensor, which is driven by electricity, is the only commercially available AE sensor [2]. However, a PZT sensor is not appropriate for use with a thin PBX material, as shown in Figure 1. In Figure 1, for the thin PBX material, the diameter is $220 \mathrm{~mm}$, and the height is $20 \mathrm{~mm}$; thus, the gap between the container and the thin PBX is $1 \mathrm{~mm}$, which is too narrow to allow a PZT AE sensor and its shielded cable to be affixed. Moreover, the PZT sensor and its cable can generate heat or sparks or short-circuit during operation, all of which pose danger to the PBX material. In Figure 1, the diameter is much larger than the height, so in this paper, the thin PBX can be considered as a 2-dimensional model.

Since the 1970s, various optical fiber sensors and signal demodulation methods have been introduced in many fields [3]. In the civil infrastructure monitoring field, optical fiber sensors can be used to detect temperature, strain, pressure, vibration, current, voltage, and gas [4]. In the biomedical field, optical fiber sensors are used for ultrasound ablation, distributed catheter shape sensing, wearable sensors, and smart textiles [5]. In materials science, optical fiber sensors are used to detect vibration, bending, and torsion [6]. 


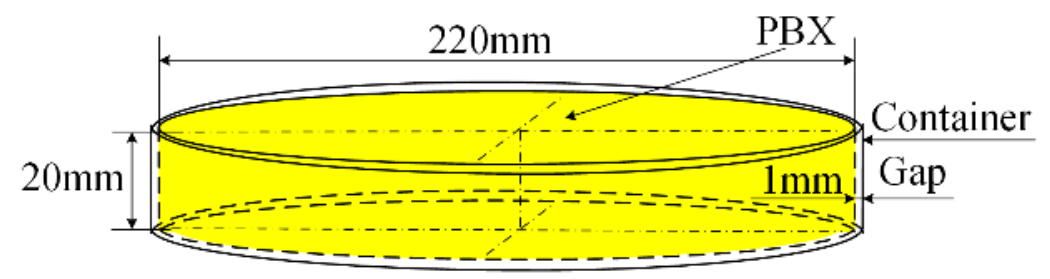

Figure 1. Thin PBX in a limited space container.

The optic fiber AE sensor is a new kind of AE sensor that has a small size, light weight, high sensitivity, anti-electromagnetic interference capability, and corrosion resistance. The fiber-optic AE sensor and its fiber pigtail do not introduce any heat or sparks during operation. These features would be large advantages in thin PBX testing.

At present, there are four kinds of fiber-optic AE sensor technologies. One type is fiber-optic F-P cavity AE technology, which has a wide measurement range and a stable system but is also difficult to produce and expensive [7]. Another type is fiber-optic ring AE technology, which has higher sensitivity than that of the other types [8,9] but also has a large fiber-optic ring $[10,11]$. The third type is fiber-optic coupler AE technology, but the sensor itself is too frail to use in practice $[12,13]$. The final type is the FBG AE sensor, which is a normal FBG sensor used for AE wave detection. The FBG AE sensor is the smallest among all types of fiber-optic AE sensors and is suitable for use in the narrow gap shown in Figure $1[14,15]$. This type of sensor can be easily produced and is low cost. Currently, research on FBG AE sensor technology focuses on detection sensitivity [16,17], frequency response range $[18,19]$, and crack location $[20,21]$.

Figure 2 shows the difference between the FBG AE sensor and the PZT AE sensor. Figure 2a shows the smallest commercial PZT AE sensor; its height is $2.4 \mathrm{~mm}$, and its diameter is $3.6 \mathrm{~mm}$. The AE wave, which is transient elastic wave, arrives at the bottom of the PZT AE sensor and oscillates inside the PZT AE sensor. During the oscillation, the AE wave will cause the PZT effect. Figure $2 b$ shows an FBG AE sensor; its length is $10 \mathrm{~mm}$, and its diameter is $0.25 \mathrm{~mm}$. The AE wave passes through the FBG grid and generates strain in the FBG AE sensor. Based on Figures 1 and 2, the FBG AE sensor is clearly smaller and more suitable for the gap with a thin PBX than the PZT AE sensor.

Thus, it can be concluded that the FBG AE sensor is more suitable for thin PBX materials than the other types of AE sensors.

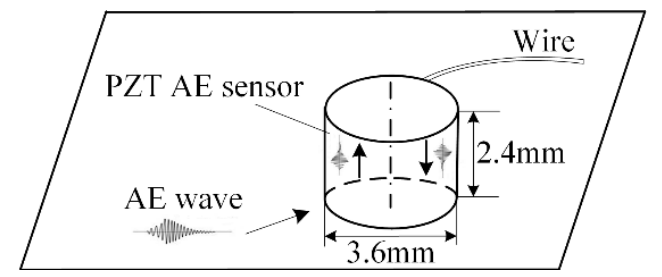

(a)

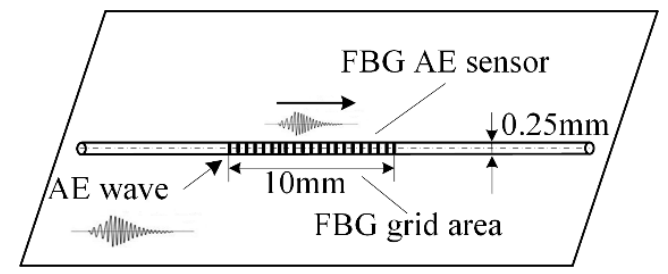

(b)

Figure 2. PZT and FBG AE sensor. (a) PZT AE sensor; (b) FBG AE sensor.

\section{Directionality of the FBG Sensor}

The central wavelength of the FBG sensor is described in (1):

$$
\lambda_{B}=2 n \Lambda
$$

where $\lambda_{B}$ is the FBG sensor central wavelength, $n$ is the effective refractive index, and $\Lambda$ is the grating period.

When an AE wave arrives at the FBG sensors, it will change $n, \Lambda$ and strain $\varepsilon$, as shown in Figure 3 . In Figure 3, the coordinate system is established. The coordinate origin $\mathrm{O}$ is the center of the FBG 
sensor. The $Y$ axis is along the axial direction of the FBG sensor. The $X$ axis is perpendicular to the $Y$ axis. $\varepsilon$ is decomposed into $\varepsilon_{x}$ and $\varepsilon_{y} . \theta$ is the angle between the AE wave propagation direction and the FBG sensor axial direction. $\theta$ is from $0^{\circ}$ to $90^{\circ}$ [22].

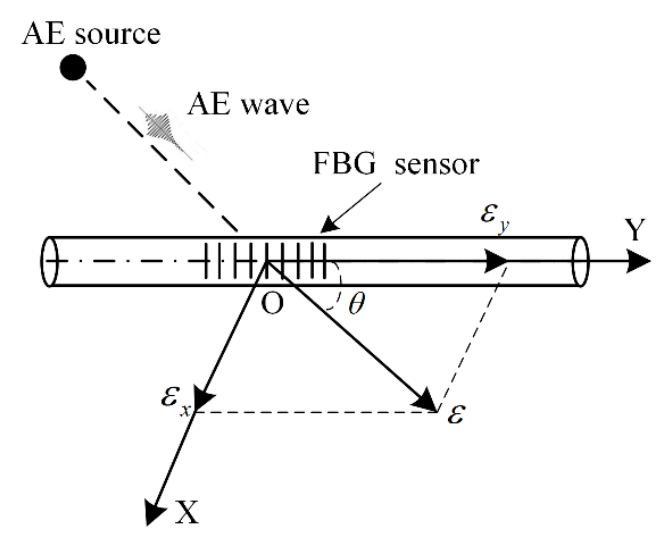

Figure 3. The coordinate system of the FBG sensor and the strain decomposition.

The quantities $n$ and $\Lambda$ are described in (2) and (3), respectively:

$$
\begin{gathered}
n=n_{0}-\frac{n_{0}^{3}}{2}\left[P_{12} \varepsilon_{y}+\left(P_{11}+P_{12}\right) \frac{\varepsilon_{x}}{2}\right] \\
\Lambda=\left(1+\varepsilon_{y}\right) \Lambda_{0}
\end{gathered}
$$

where $n_{0}$ is the initial effective refractive index, $\Lambda_{0}$ is the initial grating period, and $P_{11}$ and $P_{12}$ are Pockel's strain-optic coefficients [23].

Based on (1), $\Delta \lambda_{B}$ can be given as:

$$
\Delta \lambda_{B}=\left(\frac{\Delta n}{n_{0}}+\frac{\Delta \Lambda}{\Lambda_{0}}\right) \cdot \lambda_{B}
$$

where $\Delta \lambda_{\mathrm{B}}$ is the shift in the FBG sensor central wavelength, $\Delta n$ is the shift in the effective refractive index, and $\Delta \Lambda$ is the shift in the grating period.

When the $\mathrm{AE}$ wave is propagating along the $Y$ axis, $\varepsilon_{x}$ and $\varepsilon_{y}$ can be given as:

$$
\varepsilon_{x}=-\mu \varepsilon_{y}
$$

where $\mu$ is the Poisson ratio of the fiber.

Based on (1)-(5), $\Delta \lambda_{B 1}$ is obtained by:

$$
\begin{aligned}
\Delta \lambda_{B 1} & =\left(\frac{\Delta n}{n_{0}}+\frac{\Delta \Lambda}{\Lambda_{0}}\right) \lambda_{B} \\
& =\left(\frac{n-n_{0}}{n_{0}}+\frac{\Lambda-\Lambda_{0}}{\Lambda_{0}}\right) \lambda_{B} \\
& =\left\{1-\frac{n_{0}^{2}}{2}\left[P_{12}-\frac{\mu}{2}\left(P_{11}+P_{12}\right)\right]\right\} \lambda_{B} \varepsilon_{y} \\
& =C_{y} \lambda_{B} \varepsilon_{y} \\
& =C_{y} \lambda_{B} \varepsilon \cdot \cos \theta
\end{aligned}
$$

where $\Delta \lambda_{B 1}$ is the shift in the FBG sensor central wavelength when the AE wave is propagating along the $Y$ axis and $C_{y}=1-\frac{n_{0}^{2}}{2}\left[P_{12}-\frac{\mu}{2}\left(P_{11}+P_{12}\right)\right]$. The shift in the FBG sensor central wavelength is caused by strain generated by the AE wave. 
When the $\mathrm{AE}$ wave is propagating along the $X$ axis, $\varepsilon_{x}$ and $\varepsilon_{y}$ can be given as:

$$
-\mu \varepsilon_{x}=\varepsilon_{y}
$$

Based on (1)-(4) and (7), $\Delta \lambda_{B 2}$ is obtained by

$$
\begin{aligned}
\Delta \lambda_{B 2} & =\left\{-\mu-\frac{n_{0}^{2}}{2}\left[-\mu P_{12}+\frac{1}{2}\left(P_{11}+P_{12}\right)\right]\right\} \lambda_{B} \varepsilon_{x} \\
& =C_{x} \lambda_{B} \varepsilon_{x} \\
& =C_{x} \lambda_{B} \varepsilon \cdot \sin \theta
\end{aligned}
$$

where $\Delta \lambda_{B 2}$ is the shift in the FBG sensor central wavelength when the AE wave is propagating along the $X$ axis and $C_{x}=-\mu-\frac{n_{0}^{2}}{2}\left[-\mu P_{12}+\frac{1}{2}\left(P_{11}+P_{12}\right)\right]$.

When the $\mathrm{AE}$ wave is propagating in an arbitrary direction, $\Delta \lambda_{B}$ can be decomposed into $\Delta \lambda_{B 1}$ and $\Delta \lambda_{B 2}$. Based on Figure 3 and (6) \& (8), $\Delta \lambda_{B}$ is obtained by:

$$
\begin{gathered}
\Delta \lambda_{B}=\Delta \lambda_{B 1}+\Delta \lambda_{B 2} \\
=\left(C_{y} \cos \theta+C_{x} \sin \theta\right) \lambda_{B} \mathcal{E}
\end{gathered}
$$

Based on (9), $\Delta \lambda_{B}$ is clearly related to $\theta$.

For an FBG sensor fabricated from standard silica fiber, $n_{0}=1.45, \mu=0.17, P_{11}=0.12$, and $P_{12}=0.275$. Thus, $C_{x}=-0.328, C_{y}=0.746, \lambda_{B}>0$, and $\varepsilon>0$. Therefore, based on (9), the maximum value of $\Delta \lambda_{B}$ is reached when $\theta=0^{\circ}$, and the minimum value of $\Delta \lambda_{B}$ is reached when $\theta=90^{\circ}$.

The tunable narrowband laser detection method is used to demodulate the AE signal [24]. Figure 4 shows the tunable narrowband laser detection system and its illustrative diagram.

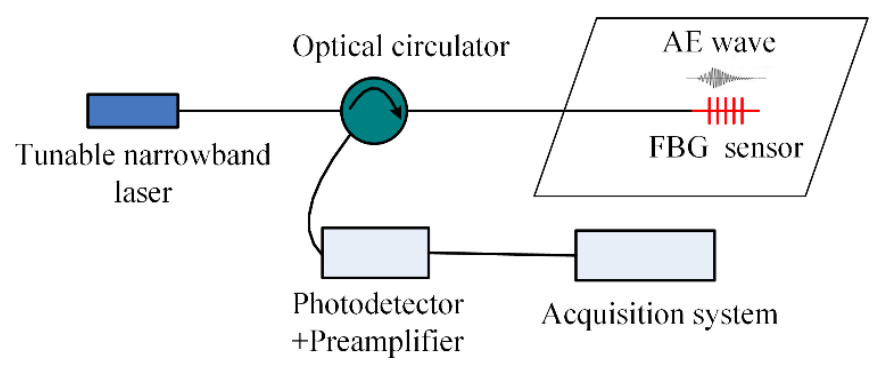

(a)

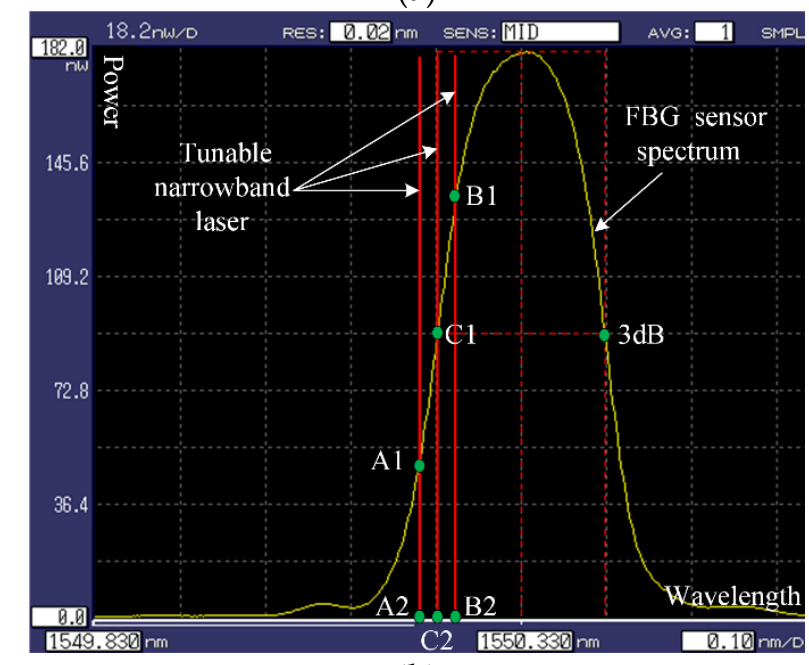

(b)

Figure 4. Tunable narrowband laser detection system and its illustrative diagram. (a) Single channel FBG sensor system; (b) Illustrative diagram. 
In Figure 4a, the tunable narrowband light penetrates the optical circulator and enters the FBG sensor. The reflected light of the FBG sensor containing the AE wave signal enters the photodetector and is then amplified by the preamplifier before finally reaching the acquisition system.

In Figure $4 \mathrm{~b}$, the bandwidth of the tunable narrowband laser is 0.0016 p.m. The bandwidth of the FBG sensor is 200 p.m., which is far greater than the tunable narrowband laser bandwidth. Thus, the spectrum of the tunable narrowband laser can be drawn as a line, as shown in Figure $4 \mathrm{~b}$. In the beginning, the output of the tunable narrowband laser is adjusted to the location of the steepest slope near the $3 \mathrm{~dB}$ point of the FBG sensor, for example, the $\mathrm{C} 1$ point in Figure $4 \mathrm{~b}$, and the reflected light power of the FBG sensor is denoted $\mathrm{C} 1 \mathrm{C} 2$. When the $\mathrm{AE}$ wave arrives at the FBG sensor, the reflected light power of the FBG sensor will change to A1A2 or B1B2. After the photodetector and the preamplifier, the output voltage of the acquisition system is proportional to $\mathrm{A} 1 \mathrm{~A} 2$ or B1B2 and is related to $\theta$, i.e., the directionality of the FBG sensor. In Figure 4, the tunable narrowband laser is the key device because it would be adjusted to fit the different FBG sensors.

The measurement results of the tunable narrowband laser detection method are dependent on spectral distortions of the attached gratings. In this paper, the spectra of the gratings show that the side-mode suppression ratio of the FBG sensor is $20 \mathrm{~dB}$, the reflectivity of the FBG sensor is $96 \%$, and the bandwidth of the FBG sensor is 200 p.m. These three parameters include an assessment of the quality of the spectra of the gratings.

\section{Experiments and Results}

\subsection{Experimental Device}

The four-channel FBG AE sensor system is shown in Figure 5, in which the tunable narrowband laser light is divided into L1, L2, L3 and L4 by coupler 1, coupler 2 and coupler 3 . The power ratio of coupler 1, coupler 2 and coupler 3 is 50:50. L1 penetrates circulator 1 and enters FBG AE sensor 1 . The reflected light with the AE wave signal from FBG AE sensor 1 is detected by photodetector 1 , and then the signal is amplified by preamplifier 1 and reaches the acquisition system. L2, L3, and L4 are identical to L1. In Figure 5, four FBG AE sensors are chosen to have the same $3 \mathrm{~dB}$ point and temperature characteristics; i.e., the four sensors exhibit the same central wavelength shift when the temperature changes. The four FBG AE sensors and the FBG temperature sensor are placed in the same temperature region. The FBG temperature sensor is used to measure the temperature changes. When the temperature changes, the signal from the FBG temperature sensor is sent to the temperature demodulator system and the computer. Thus, when the temperature changes, the computer can adjust the output of the tunable narrowband laser to the location of the steepest slope near the $3 \mathrm{~dB}$ point of the four FBG AE sensors, as shown in Figure $4 \mathrm{~b}$. 


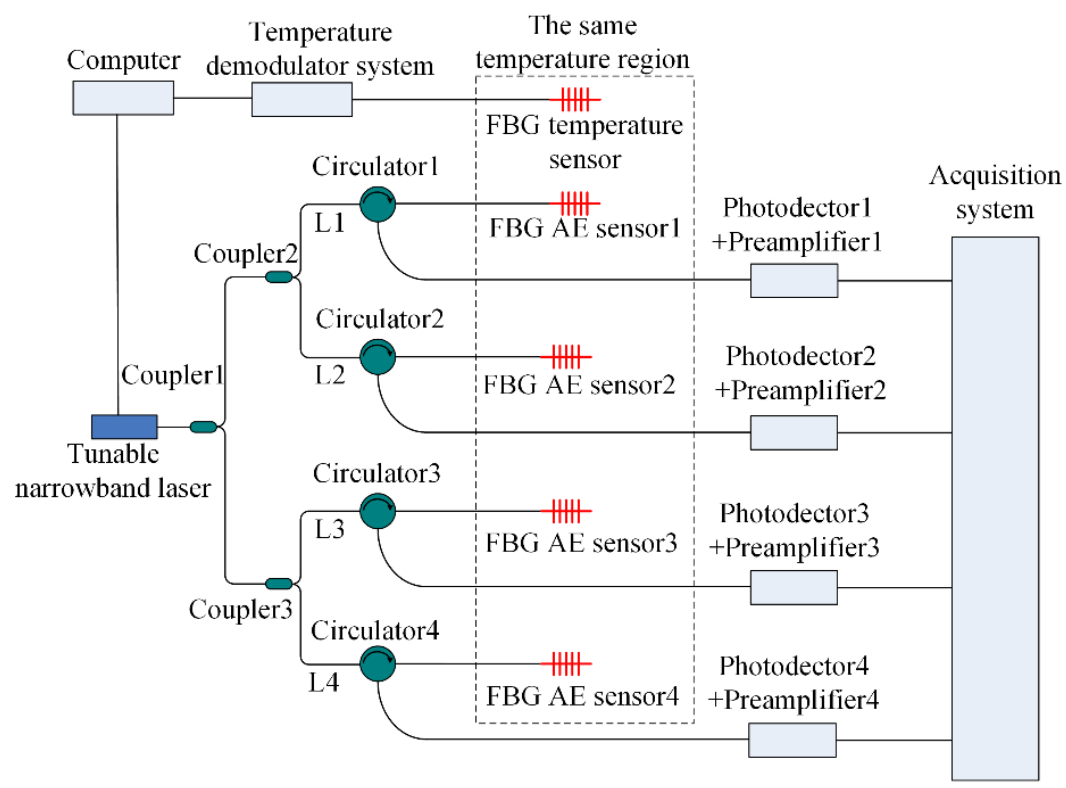

Figure 5. Four-channel FBG AE sensor system.

Based on Figure 5, the four-channel FBG AE sensor system is built, as shown in Figure 6. The tunable narrowband laser is model TSL-510 from Santec Corporation (Aichi, Japan); the output wavelength is $1550 \mathrm{~nm}$, and the power is $8 \mathrm{~mW}$. The photodetector is model 2117 from New Focus Company (Irvine, CA, USA). The preamplifier is model 2/4/6 from Physical Acoustics Corporation (Princeton Junction, NJ, USA). The acquisition device is model Express 8 from Physical Acoustics Corporation. The computer is model IPC-610L from ADVANTECH Company (Taiwan, China). The temperature demodulator system includes model FBGA-S-1525-1605-FA from BaySpec Corporation (San Jose, CA, USA) and model ASE-CL-M2 from Top Photonics Corporation (Beijing, China).

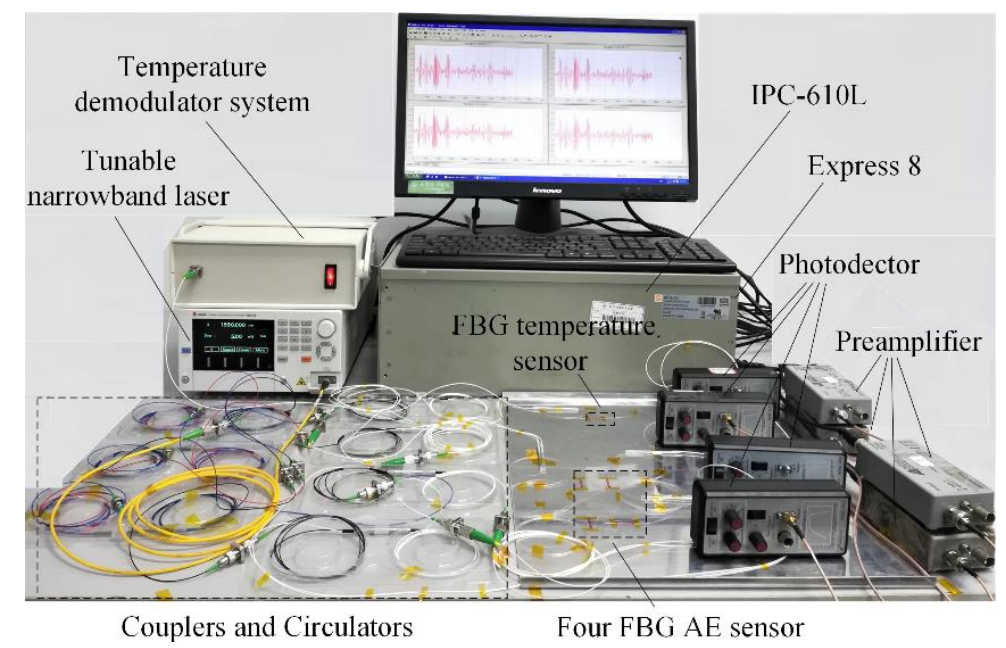

Figure 6. FBG AE system setup.

\subsection{FBG AE Sensor Directionality Experiment}

The FBG AE sensor is glued onto the surface of a thin PBX with cyanoacrylate, as shown in Figure 7. 


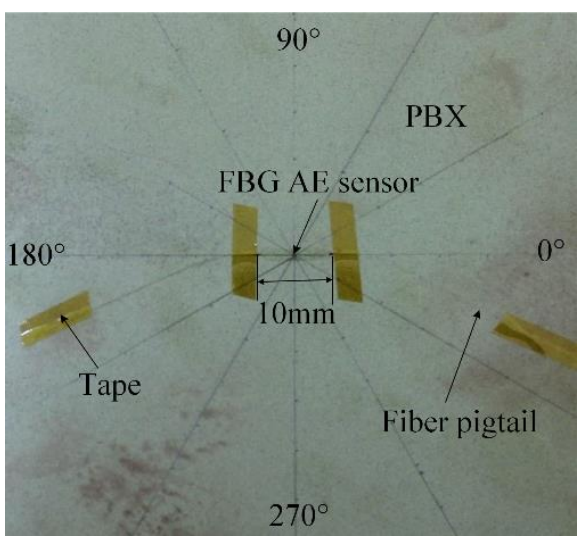

(a)

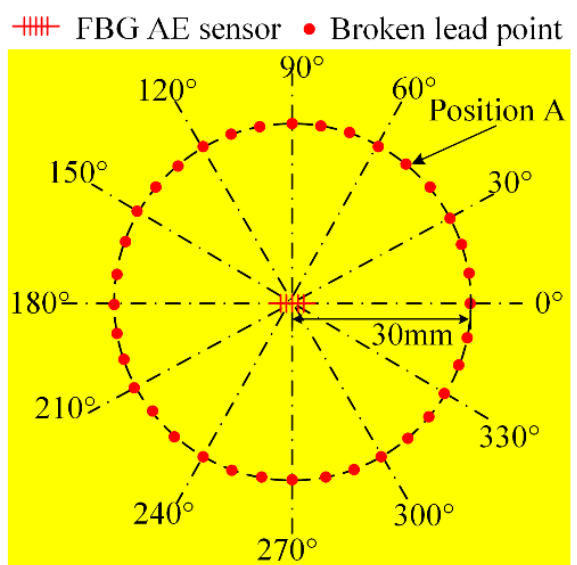

(b)

Figure 7. FBG AE sensor directionality experiment. (a) Photograph of the FBG AE sensor on the PBX surface; (b) FBG AE sensor experiment and broken lead point.

Figure 7a shows a photograph of the actual FBG AE sensor setup. In Figure 7a, the FBG AE sensor whose length is $10 \mathrm{~mm}$ is pasted along the horizontal direction. Along the horizontal directions, the angles are $0^{\circ}$ and $180^{\circ}$. Along the perpendicular directions, the angles are $90^{\circ}$ and $270^{\circ}$.

The Hsu-Nielsen broken lead method, is used in the experiments, where the pencil lead diameter is $0.5 \mathrm{~mm}$, the hardness is $2 \mathrm{H}$ and the length is $3.0 \mathrm{~mm}$. When this kind of pencil lead is broken on the surface of PBX materials, it will produce a standard AE signal. That signal can be used to simulate the AE signal at the breaking lead position $[25,26]$. For example, when the broken lead is at position A in Figure $7 \mathrm{~b}$, it will simulate an AE signal conducted at position A.

Figure $7 \mathrm{~b}$ shows the location of broken lead points. In Figure $7 \mathrm{~b}$, the broken lead points are located at 36 different directions: $0^{\circ}, 10^{\circ}, 20^{\circ}, 30^{\circ}, 40^{\circ}, \ldots, 330^{\circ}, 340^{\circ}$, and $350^{\circ}$. In each direction, the distance between the center of the FBG AE sensor and the broken lead points is $30 \mathrm{~mm}$. The pencil lead is broken three times at each broken lead point. Using one channel of the FBG AE sensor system, as shown in Figure 5, the threshold voltage of the acquisition system is set to $100 \mu \mathrm{V}(40 \mathrm{~dB})$, and then the signal amplitude and arrival time $T$ are obtained. The average signal amplitude of three broken leads at each point is shown in Figure 8.

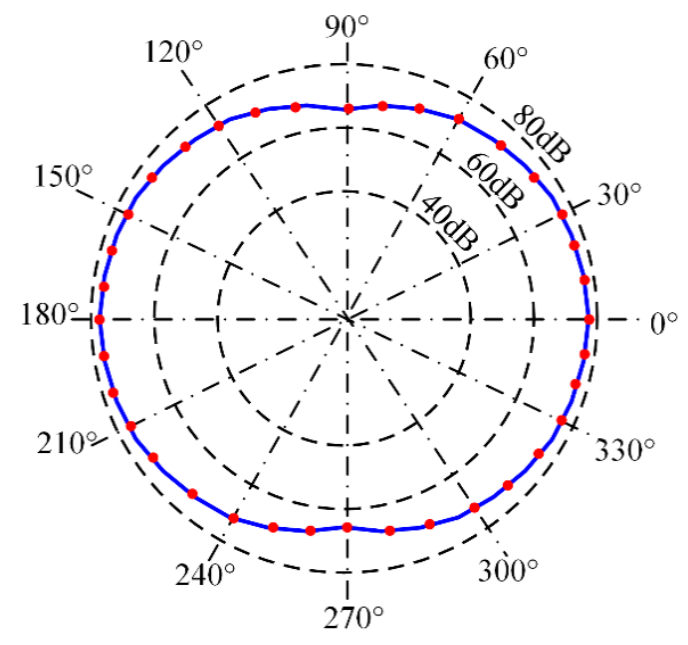

Figure 8. Polar diagram of the FBG AE sensor signal amplitude. 
In Figure 8, the dot-dashed line shows the angle from $0^{\circ}$ to $330^{\circ}$, and the dashed line shows the amplitude from $40 \mathrm{~dB}$ to $80 \mathrm{~dB}$. The red round dot is the signal amplitude along the directions of $0^{\circ}$, $10^{\circ}, 20^{\circ}, 30^{\circ}, 40^{\circ}, \ldots, 330^{\circ}, 340^{\circ}$, and $350^{\circ}$.

In Figure 8, by connecting the red round dots, the blue curve is obtained. Clearly, the blue curve exhibits longitudinal and horizontal symmetry. Thus, the curve from $0^{\circ}$ to $90^{\circ}$ can be studied as an example. Moreover, other areas are similar to it.

In Figure 8, the signal amplitude is clearly dependent on angle $\theta$. The highest signal amplitude is observed along the $0^{\circ}$ direction, and the lowest amplitude is observed along the $90^{\circ}$ direction. Therefore, it verified (9) and the directionality of the FBG AE sensor.

The average arrival time $T$ of three broken leads in the range from $0^{\circ}$ to $90^{\circ}$ is shown in Table 1 .

Table 1. Angle $\theta$ and arrival time $T$ of the FBG AE sensor.

\begin{tabular}{cc}
\hline Angle $\boldsymbol{\theta}$ & Arrival Time $\boldsymbol{T} / \boldsymbol{\mu s}$ \\
\hline $0^{\circ}$ & 2.30 \\
$10^{\circ}$ & 2.40 \\
$20^{\circ}$ & 2.60 \\
$30^{\circ}$ & 2.65 \\
$40^{\circ}$ & 2.90 \\
$50^{\circ}$ & 3.55 \\
$60^{\circ}$ & 4.20 \\
$70^{\circ}$ & 5.40 \\
$80^{\circ}$ & 6.20 \\
$90^{\circ}$ & 8.50 \\
\hline
\end{tabular}

Based on Table 1 and the curve fitting tool in MATLAB software, the relationship between angle $\theta$ and time $T$ is given as:

$$
T(\theta)=-2.623 \times 10^{-8} \cdot \theta^{4}+1.429 \times 10^{-5} \cdot \theta^{3}-4.542 \times 10^{-4} \cdot \theta^{2}+1.314 \times 10^{-2} \cdot \theta+2.3
$$

where $T(\theta)$ is a 4 th order polynomial function. Based on (10) and Table 1, Figure 9 can be obtained.

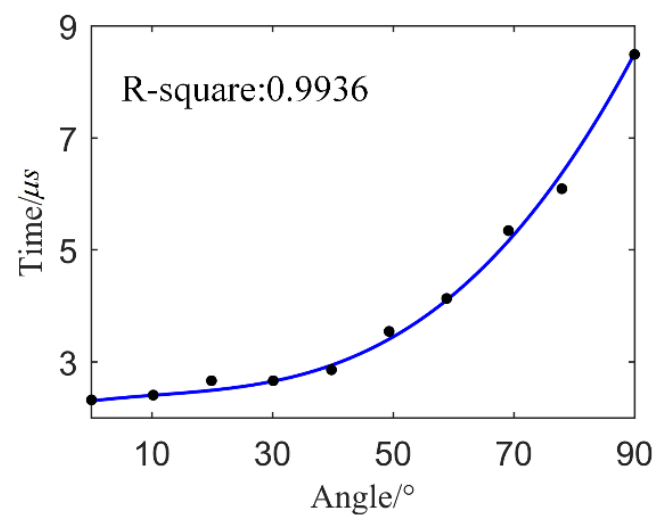

Figure 9. Polynomial function $T(\theta)$.

In Figure 9, the black points denote the real arrival time based on Table 1, and the blue curve is $T(\theta)$ based on (10); the R-square between them is 0.9936 . Thus, the blue curve is found to match the black points very well. The 4 th-order polynomial function $T(\theta)$ may not be the best one, but in this paper, it works well.

In Figure 9, the signal arrival time increases with the angle, thus verifying the directionality of the FBG AE sensor. Based on traditional AE location theory, the signal arrival time determines the location [27]. In other words, the directionality of the FBG AE sensor will influence the location. 


\subsection{AE Source Location Experiment on a Thin PBX Sample}

Figure 10a shows a photograph of the sensors on the setup and the angles for the source location experiment. In Figure 10a, FBG AE sensor 1 and sensor 3 are pasted along the horizontal direction, and sensor 2 and sensor 4 are pasted along the perpendicular direction. Along the horizontal directions, the angles are $0^{\circ}$ and $180^{\circ}$. The $45^{\circ}$ direction is between the $0^{\circ}$ and $90^{\circ}$ directions. Moreover, along the perpendicular directions, the angles are $90^{\circ}$ and $270^{\circ}$. The $225^{\circ}$ direction is between the $180^{\circ}$ and $270^{\circ}$ directions.

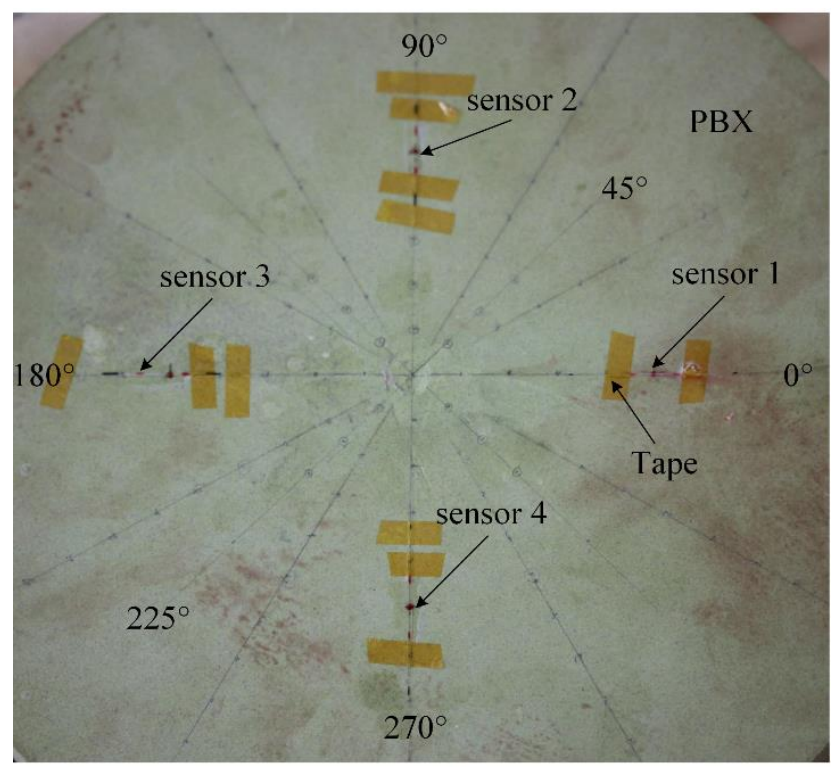

(a)

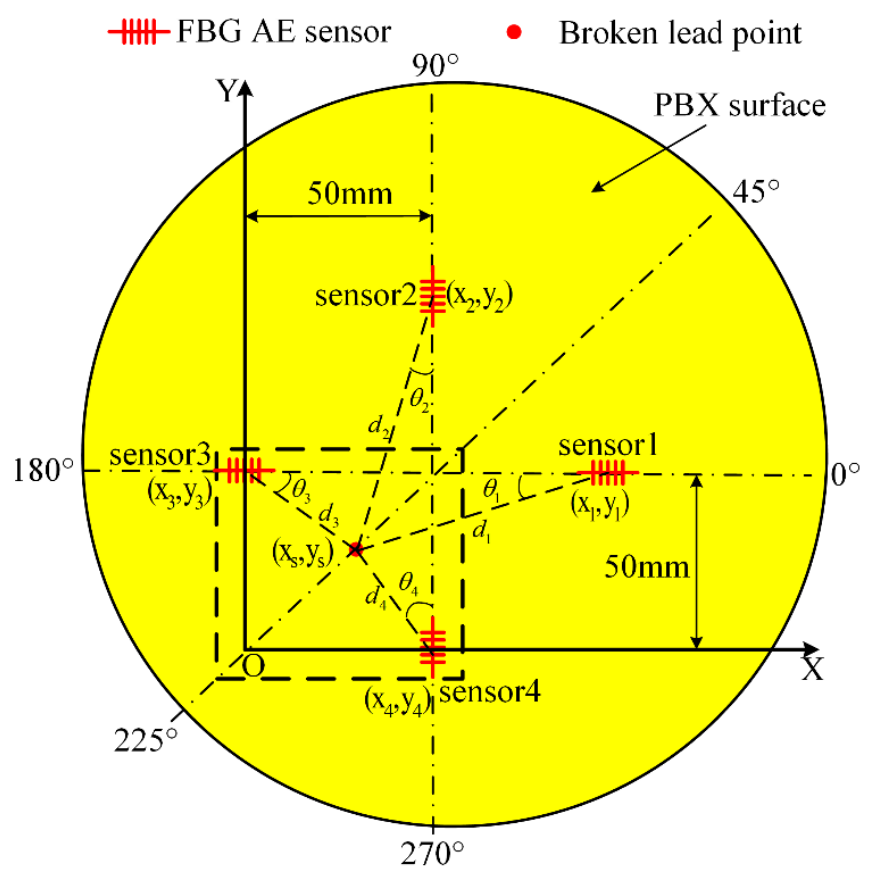

(b)

Figure 10. FBG AE source location experiment on a thin PBX sample. (a) Photograph of four sensors on the PBX surface; (b) source location experiment of a broken lead point.

Figure 10b shows the FBG AE source location experiment on a thin PBX sample and the corresponding source location experiment. In Figure 10b, there are four FBG AE sensors: sensor 1, 
sensor 2, sensor 3 and sensor 4 . The offset between the center of sensor 1 and the $X$ axis is $50 \mathrm{~mm}$, and the offset between the center of sensor 2 and the $Y$ axis is $50 \mathrm{~mm}$; the same is true for sensor 3 and sensor $4 . \theta_{1}, \theta_{2}, \theta_{3}$ and $\theta_{4}$ are the angles between the propagation direction of the AE wave and the axial direction of the FBG AE sensors. The coordinate system is established on the surface of the thin PBX sample. The $Y$ axis of the coordinate system is along the perpendicular direction passing through the center of FBG AE sensor 3. The $X$ axis of the coordinate system is along the horizontal direction passing through the center of FBG AE sensor 4. Moreover, the origin $\mathrm{O}$ of the coordinate system is the intersection of the $X$ axis and the $Y$ axis. In the coordinate system, the center positions of the four FBG AE sensors are $\left(x_{1}, y_{1}\right),\left(x_{2}, y_{2}\right),\left(x_{3}, y_{3}\right)$ and $\left(x_{4}, y_{4}\right)$; the position of the broken lead is $\left(x_{s}, y_{s}\right)$. Four sensors are placed radially clocked every 90 degrees in azimuth; this arrangement can guarantee that the broken lead signal in $\left(x_{s}, y_{s}\right)$ is detected by all the sensors.

The point of the AE source in the traditional location method can be calculated as:

$$
\begin{aligned}
& T_{1}-T_{2}=\left(\sqrt{\left(x_{1}-x_{s}^{\prime}\right)^{2}+\left(y_{1}-y_{s}^{\prime}\right)^{2}}-\sqrt{\left(x_{2}-x_{s}^{\prime}\right)^{2}+\left(y_{2}-y_{s}^{\prime}\right)^{2}}\right) / v \\
& T_{1}-T_{3}=\left(\sqrt{\left(x_{1}-x_{s}^{\prime}\right)^{2}+\left(y_{1}-y_{s}^{\prime}\right)^{2}}-\sqrt{\left(x_{3}-x_{s}^{\prime}\right)^{2}+\left(y_{3}-y_{s}^{\prime}\right)^{2}}\right) / v \\
& T_{1}-T_{4}=\left(\sqrt{\left(x_{1}-x_{s}^{\prime}\right)^{2}+\left(y_{1}-y_{s}^{\prime}\right)^{2}}-\sqrt{\left(x_{4}-x_{s}^{\prime}\right)^{2}+\left(y_{4}-y_{s}^{\prime}\right)^{2}}\right) / v
\end{aligned}
$$

where $T_{1}, T_{2}, T_{3}$ and $T_{4}$ are the arrival times for FBG AE sensor 1, sensor 2, sensor 3 and sensor 4, respectively. $v$ is the velocity of the AE wave. $\left(x_{s}^{\prime}, y_{s}^{\prime}\right)$ is the point of the AE source calculated by the traditional location method [27].

For the location experiment shown in Figure 10, $T_{1}=0.3060 \mathrm{~ms}, T_{2}=0.3065 \mathrm{~ms}, T_{3}=0.3800 \mathrm{~ms}$, $T_{4}=0.3073 \mathrm{~ms}, v=1145(\mathrm{~mm} / \mathrm{ms}),\left(x_{1}, y_{1}\right)=(100.0 \mathrm{~mm}, 50.0 \mathrm{~mm}),\left(x_{2}, y_{2}\right)=(50.0 \mathrm{~mm}, 100.0 \mathrm{~mm})$, $\left(x_{3}, y_{3}\right)=(0.0 \mathrm{~mm}, 50.0 \mathrm{~mm})$, and $\left(x_{4}, y_{4}\right)=(50.0 \mathrm{~mm}, 0.0 \mathrm{~mm})$.

Calculating (11) and (12) by the least squares method in MATLAB software, the numerical error for the least square method is less than $1.0 \times 10^{-6}$. The result is given by:

$$
\left(x_{s 1}^{\prime}, y_{s 1}^{\prime}\right)=(51.1 \mathrm{~mm}, 51.0 \mathrm{~mm}) \text { units not italic font }
$$

where $\left(x_{s 1}^{\prime}, y_{s 1}^{\prime}\right)$ is the point of the AE source calculated by (11) and (12).

Performing the same calculation for (12) and (13) as that described above, the result is:

$$
\left(x_{s 2}^{\prime}, y_{s 2}^{\prime}\right)=(51.1 \mathrm{~mm}, 50.3 \mathrm{~mm})
$$

where $\left(x_{s 2}^{\prime}, y_{s 2}^{\prime}\right)$ is the point of the AE source calculated by (12) and (13).

Upon performing the same calculation of (11) and (13) as above, the result is:

$$
\left(x_{s 3}^{\prime}, y_{s 3}^{\prime}\right)=(50.5 \mathrm{~mm}, 51.1 \mathrm{~mm})
$$

where $\left(x_{s 3}^{\prime}, y_{s 3}^{\prime}\right)$ is the point of the AE source calculated by (11) and (13).

Based on (14)-(16), the final average location of the AE source is:

$$
\left(x_{s}^{\prime}, y_{s}^{\prime}\right)=(50.9 \mathrm{~mm}, 50.1 \mathrm{~mm})
$$

The error $E_{1}$ between $\left(x_{s}, y_{s}\right)=(28.8 \mathrm{~mm}, 28.8 \mathrm{~mm})$ and $\left(x_{s}^{\prime}, y_{s}^{\prime}\right)=(50.9 \mathrm{~mm}, 50.8 \mathrm{~mm})$ can be obtained, as shown in Figure 11. Figure 11 shows an enlarged view of the area defined by the dashed line in Figure 10:

$$
\begin{aligned}
E_{1} & =\sqrt{\left(x_{s}-x_{s}^{\prime}\right)^{2}+\left(y_{s}-y_{s}^{\prime}\right)^{2}} \\
& =\sqrt{(28.8-50.9)^{2}+(28.8-50.8)^{2}} \\
& =31.2 \mathrm{~mm}
\end{aligned}
$$




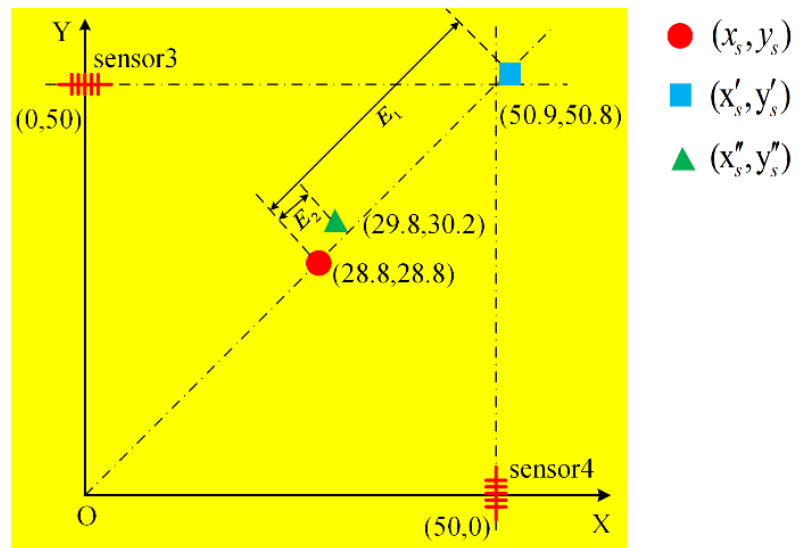

Figure 11. The result of the location experiment.

In Figure 11, there is a large error $E_{1}$ between $\left(x_{s}, y_{s}\right)$ and $\left(x_{s}^{\prime}, y_{s}^{\prime}\right)$. The reason for this large error lies in the directionality of the FBG AE sensor. Therefore, the traditional location method (11)-(13) is not suitable for the FBG AE sensor; as a result, the location result obtained using the traditional method is inaccurate.

Based on the result of the FBG AE sensor directionality experiment, the time coefficient method is proposed to locate the source of the broken lead point. For the FBG AE sensor glued onto the surface of the thin PBX sample, the time coefficient $h(\theta)$ is introduced to calibrate the location. Based on (10), $h(\theta)$ can be described as:

$$
\begin{aligned}
h(\theta) & =\frac{T\left(\theta=0^{\circ}\right)}{T(\theta)} \\
& =\frac{2.3}{-2.623 \times 10^{-8} \cdot \theta^{4}+1.429 \times 10^{-5} \cdot \theta^{3}-4.542 \times 10^{-4} \cdot \theta^{2}+1.314 \times 10^{-2} \cdot \theta+2.3}
\end{aligned}
$$

In Figure $10, \theta_{1}, \theta_{2}, \theta_{3}$ and $\theta_{4}$ can be calculated as follows:

$$
\begin{aligned}
& \theta_{1}=\arctan \left(\frac{y_{1}-y_{s}}{x_{1}-x_{s}}\right) \\
& \theta_{2}=\arctan \left(\frac{x_{2}-x_{s}}{y_{2}-y_{s}}\right) \\
& \theta_{3}=\arctan \left(\frac{y_{3}-y_{s}}{x_{s}-x_{3}}\right) \\
& \theta_{4}=\arctan \left(\frac{x_{4}-x_{s}}{y_{s}-y_{4}}\right)
\end{aligned}
$$

$h\left(\theta_{1}\right), h\left(\theta_{2}\right), h\left(\theta_{3}\right)$ and $h\left(\theta_{4}\right)$ can be obtained as follows:

$$
\begin{aligned}
& h\left(\theta_{1}\right)=\frac{2.3}{-2.623 \times 10^{-8} \cdot \theta_{1}^{4}+1.429 \times 10^{-5} \cdot \theta_{1}^{3}-4.542 \times 10^{-4} \cdot \theta_{1}^{2}+1.314 \times 10^{-2} \cdot \theta_{1}+2.3} \\
& h\left(\theta_{2}\right)=\frac{2.3}{-2.623 \times 10^{-8} \cdot \theta_{2}{ }^{4}+1.429 \times 10^{-5} \cdot \theta_{2}{ }^{3}-4.542 \times 10^{-4} \cdot \theta_{2}{ }^{2}+1.314 \times 10^{-2} \cdot \theta_{2}+2.3} \\
& h\left(\theta_{3}\right)=\frac{2.3}{-2.623 \times 10^{-8} \cdot \theta_{3}{ }^{4}+1.429 \times 10^{-5} \cdot \theta_{3}^{3}-4.542 \times 10^{-4} \cdot \theta_{3}{ }^{2}+1.314 \times 10^{-2} \cdot \theta_{3}+2.3} \\
& h\left(\theta_{4}\right)=\frac{2.3}{-2.623 \times 10^{-8} \cdot \theta_{4}^{4}+1.429 \times 10^{-5} \cdot \theta_{4}^{3}-4.542 \times 10^{-4} \cdot \theta_{4}^{2}+1.314 \times 10^{-2} \cdot \theta_{4}+2.3}
\end{aligned}
$$


Based on (19)-(27), the point of the AE source in the time coefficient location method can be given as follows:

$$
\begin{aligned}
& T_{1} \cdot h\left(\theta_{1}\right)-T_{2} \cdot h\left(\theta_{2}\right)=\left(\sqrt{\left(x_{1}-x_{s}^{\prime \prime}\right)^{2}+\left(y_{1}-y_{s}^{\prime \prime}\right)^{2}}-\sqrt{\left(x_{2}-x_{s}^{\prime \prime}\right)^{2}+\left(y_{2}-y_{s}^{\prime \prime}\right)^{2}}\right) / v \\
& T_{1} \cdot h\left(\theta_{1}\right)-T_{3} \cdot h\left(\theta_{3}\right)=\left(\sqrt{\left(x_{1}-x_{s}^{\prime \prime}\right)^{2}+\left(y_{1}-y_{s}^{\prime \prime}\right)^{2}}-\sqrt{\left(x_{3}-x_{s}^{\prime \prime}\right)^{2}+\left(y_{3}-y_{s}^{\prime \prime}\right)^{2}}\right) / v \\
& T_{1} \cdot h\left(\theta_{1}\right)-T_{4} \cdot h\left(\theta_{4}\right)=\left(\sqrt{\left(x_{1}-x_{s}^{\prime \prime}\right)^{2}+\left(y_{1}-y_{s}^{\prime \prime}\right)^{2}}-\sqrt{\left(x_{4}-x_{s}^{\prime \prime}\right)^{2}+\left(y_{4}-y_{s}^{\prime \prime}\right)^{2}}\right) / v
\end{aligned}
$$

where $h\left(\theta_{1}\right), h\left(\theta_{2}\right), h\left(\theta_{3}\right)$ and $h\left(\theta_{4}\right)$ are the time coefficients of $T_{1}, T_{2}, T_{3}$ and $T_{4}$, respectively. $\left(x_{s}^{\prime \prime}, y_{s}^{\prime \prime}\right)$ is the point of the AE source calculated by the time coefficient location method.

Calculating (28) and (29) by the least squares method in MATLAB software, the numerical error for the least squares method is less than $1.0 \times 10^{-6}$; the result is given as follows:

$$
\left(x_{s 1}^{\prime \prime}, y_{s 1}^{\prime \prime}\right)=(29.3 \mathrm{~mm}, 30.1 \mathrm{~mm})
$$

where $\left(x_{s 1}^{\prime \prime}, y_{s 1}^{\prime \prime}\right)$ is the point of the AE source calculated by (28) and (29).

Performing the same calculation of (29) and (30) as that described above, the result is

$$
\left(x_{s 2}^{\prime \prime}, y_{s 2}^{\prime \prime}\right)=(30.1 \mathrm{~mm}, 29.8 \mathrm{~mm})
$$

where $\left(x_{s 2}^{\prime \prime}, y_{s 2}^{\prime \prime}\right)$ is the point of the AE source calculated by (29) and (30).

Performing the same calculation of (28) and (30) as that described above, the result is

$$
\left(x_{s 3}^{\prime \prime}, y_{s 3}^{\prime \prime}\right)=(29.9 \mathrm{~mm}, 30.8 \mathrm{~mm})
$$

where $\left(x_{s 3}^{\prime \prime}, y_{s 3}^{\prime \prime}\right)$ is the point of the AE source calculated by (28) and (30).

Based on (31)-(33), the average location of the AE source is

$$
\left(x_{s}^{\prime \prime}, y_{s}^{\prime \prime}\right)=(29.8 \mathrm{~mm}, 30.2 \mathrm{~mm})
$$

The error $E_{2}$ between $\left(x_{s}, y_{s}\right)=(28.8 \mathrm{~mm}, 28.8 \mathrm{~mm})$ and $\left(x_{s}^{\prime \prime}, y_{s}^{\prime \prime}\right)=(29.8 \mathrm{~mm}, 30.2 \mathrm{~mm})$ can be obtained, as shown in Figure 11.

$$
\begin{aligned}
E_{2} & =\sqrt{\left(x_{s}-x_{s}^{\prime \prime}\right)^{2}+\left(y_{s}-y_{s}^{\prime \prime}\right)^{2}} \\
& =\sqrt{(28.8-29.8)^{2}+(28.8-30.2)^{2}} \\
& =1.7 \mathrm{~mm}
\end{aligned}
$$

In Figure 11, error $E_{2}$ is less than error $E_{1}$. For the FBG AE sensor glued onto the surface of PBX, the time coefficient location method of (28)-(30) is clearly better than the traditional location method of (11)-(13).

The experiments were repeated six times at different positions, as shown in Figure 12.

In Figure 12, No. 1, No. 2, No. 3, No. 4, No. 5 and No. 6 are the locations of the broken lead experiments. In the coordinate system, the locations of No. 1, No. 2, No. 3, No. 4, No. 5 and No. 6 are $(28.8,28.8),(35.9,35.9),(42.9,42.9),(40.0,50.0),(30.0,50.0)$ and $(20.0,50.0)$, respectively. 


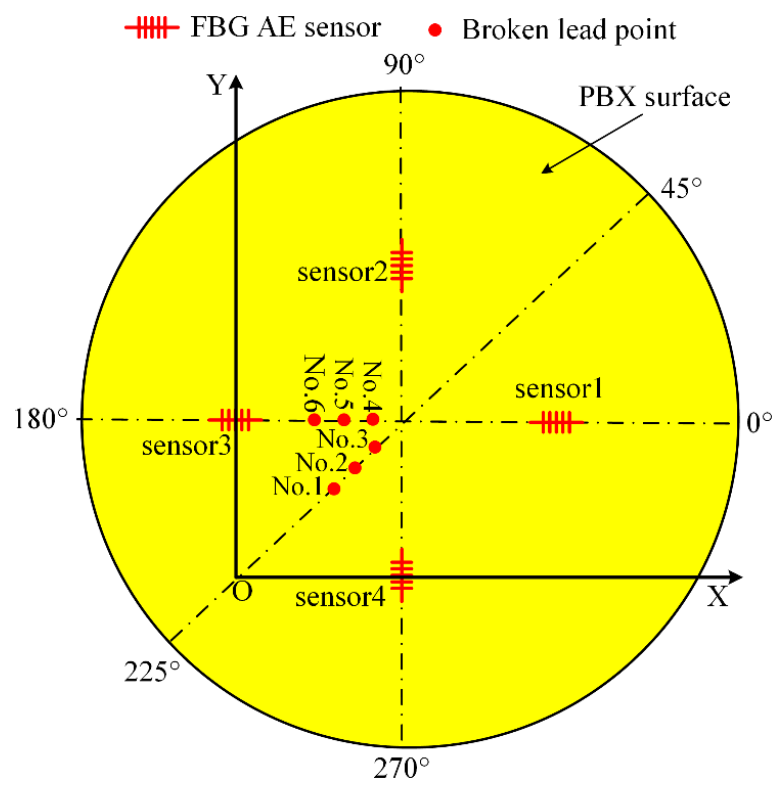

Figure 12. Locations of the broken lead.

The arrival time of the four FBG AE sensors recorded in the experiments repeated six times are shown in Table 2.

Table 2. Arrival time.

\begin{tabular}{ccccc}
\hline Experiment Locations & $\mathbf{T}_{\mathbf{1}} / \mathbf{m s}$ & $\mathbf{T}_{\mathbf{2}} / \mathbf{m s}$ & $\mathbf{T}_{\mathbf{3}} / \mathbf{m s}$ & $\mathbf{T}_{\mathbf{4}} / \mathbf{m s}$ \\
\hline No. 1 & 0.3360 & 0.3365 & 0.3380 & 0.3373 \\
No. 2 & 0.2065 & 0.2080 & 0.1890 & 0.1875 \\
No. 3 & 0.4750 & 0.4755 & 0.4660 & 0.4653 \\
No. 4 & 0.3602 & 0.3700 & 0.3505 & 0.3703 \\
No. 5 & 0.2505 & 0.2680 & 0.2365 & 0.2665 \\
No. 6 & 0.3468 & 0.3790 & 0.3003 & 0.3770 \\
\hline
\end{tabular}

The results of the location calculations are shown in Table 3.

Table 3. Location results and their errors.

\begin{tabular}{cccccc}
\hline Experiment Locations & $\begin{array}{c}\left(x_{s}, y_{s}\right) \\
/ \mathbf{m m}\end{array}$ & $\begin{array}{c}\left(x_{s}^{\prime}, y_{s}^{\prime}\right) \\
/ \mathbf{m m}\end{array}$ & $\begin{array}{c}E_{\mathbf{1}} \\
/ \mathbf{m m}\end{array}$ & $\begin{array}{c}\left(x_{s}^{\prime \prime}, y_{s}^{\prime \prime}\right) \\
/ \mathbf{m m}\end{array}$ & $\begin{array}{c}E_{\mathbf{2}} \\
/ \mathbf{m m}\end{array}$ \\
\hline No. 1 & $(28.8,28.8)$ & $(50.9,50.8)$ & 31.2 & $(29.8,30.2)$ & 1.7 \\
No. 2 & $(35.9,35.9)$ & $(39.8,38.0)$ & 4.5 & $(35.0,36.6)$ & 1.2 \\
No. 3 & $(42.9,42.9)$ & $(44.9,44.4)$ & 2.5 & $(44.3,43.9)$ & 1.7 \\
No. 4 & $(40.0,50.0)$ & $(49.5,50.0)$ & 9.5 & $(39.5,48.7)$ & 1.4 \\
No. 5 & $(30.0,50.0)$ & $(48.8,47.0)$ & 19.0 & $(29.7,48.4)$ & 1.7 \\
No. 6 & $(20.0,50.0)$ & $(70.4,71.7)$ & 55.0 & $(21.2,49.5)$ & 1.3 \\
\hline
\end{tabular}

In Table 3, the error $E_{1}$ is dependent on the position, i.e., lower error for position No. 3 and higher error for position No. 6. The different results for the different positions correspond to different $\theta_{1}, \theta_{2}$, $\theta_{3}$ and $\theta_{4}$, as shown in Figure $10 \mathrm{~b}$. The greater the deviation of the four angles is, the greater is the influence of the FBG AE sensor directivity, and the greater the final error.

In Table 3, the location errors $E_{1}$ calculated by the traditional location method are $31.2 \mathrm{~mm}, 4.5 \mathrm{~mm}$, $2.5 \mathrm{~mm}, 9.5 \mathrm{~mm}, 19.0 \mathrm{~mm}$, and $55.0 \mathrm{~mm}$. The location errors $E_{2}$ calculated by the time coefficient method are $1.7 \mathrm{~mm}, 1.2 \mathrm{~mm}, 1.7 \mathrm{~mm}, 1.4 \mathrm{~mm}, 1.7 \mathrm{~mm}$, and $1.3 \mathrm{~mm}$. Obviously, the location errors of the time coefficient method are much smaller than those of the traditional location method. 


\section{Summary and Conclusions}

In this paper, the novelty lies in three areas. The first is the time coefficient location method based on the directionality of the FBG AE sensor. The second is the establishment of a four-channel FBG $\mathrm{AE}$ device to perform the experiments. The last is FBG AE sensor experiments conducted on thin PBX materials.

In the FBG AE sensor directional experiment, the amplitude and arrival time detected by the FBG AE sensor were found to be related to the angle between the propagation direction of the $\mathrm{AE}$ wave and the axial direction of the FBG AE sensors. Based on the directionality of the FBG AE sensor, the time coefficient location method was proposed to correct the time detected by the FBG AE sensors. The results of the location experiments on the surface of thin PBX showed that the accuracy of the sensor is good.

For the FBG AE sensors mentioned in the paper, the threshold voltage is $40 \mathrm{~dB}$. As a result, smaller actual defects/cracks could not be detected. Only when the amplitude of an actual crack is greater than $40 \mathrm{~dB}$ can the $\mathrm{AE}$ wave be collected by the sensors. For the time coefficient location method, only the surface cracks limited to the region near the center of thin PBX could be located. Otherwise, the method must be changed. Thus, we believe that the FBG AE sensors and the time coefficient location method need further research before their fully mature application in tasks involving PBX materials. In the paper, usage of a multiple FBG-based system for AE measurements on PBX increases the complexity and installation and equipment costs. However, it could also improve the safety of PBX materials, which are the main component of detonation devices in nuclear bombs and the main part of explosive bolts for multistage heavy launch vehicles and large satellite solar wings. Therefore, any investment in the safety of PBX materials would help improve the safety of those important strategic weapons.

Author Contributions: T.F. designed the experiments. P.W. conceived the sensors and analyzed the data. X.H. and Q.L. conducted the experiments. All authors contributed to the preparation of the manuscript.

Funding: This research was funded by the National Science Foundation of China grant number 11604309.

Acknowledgments: This work was supported in part by the National Science Foundation of China (Grant No. 11604309), the Institute of Chemical Materials, China Academy of Engineering Physics, and the Ministry of Industry and Information Technology of the People's Republic of China.

Conflicts of Interest: The authors declare no conflict of interest.

\section{Abbreviations}

The following abbreviations are used in this manuscript.

$\mathrm{AE} \quad$ acoustic emission

PBX polymer bonded explosives

PZT piezoelectric

FBG fiber Bragg grating

\section{References}

1. Shen, G.T. Acoustic Emission Technology and Application, 1st ed.; Science Press: Beijing, China, 2015; pp. 1-5, ISBN 978-7-03-044522-3.

2. Garcia-Souto, J.A.; Lamela-River, H. High resolution $(<1 \mathrm{~nm})$ interferometric fiber-optic sensor of vibrations in high-power transformers(. Opt. Express 2006, 14, 9679-9686. [CrossRef] [PubMed]

3. Lee, B. Review of Present Status of Optical Fiber Sensors. Opt. Fiber Technol. 2003, 9, 57-79. [CrossRef]

4. Bao, X.Y.; Chen, L. Recent progress in distributed fiber optic sensors. Sensors 2012, 12, 8601-8639. [CrossRef] [PubMed]

5. Tosi, D.; Schena, E.; Molardi, C.; Korganbayev, S. Fiber optic sensors for sub-centimeter spatially resolved measurements: Review and biomedical applications. Opt. Fiber Technol. 2018, 43, 6-19. [CrossRef]

6. Chen, J.J.; Liu, B.; Zhang, H. Review of fiber Bragg grating sensor technology. Front. Optoelectron. China 2011, 4, 204-212. [CrossRef] 
7. De Oliveira, R.; Ramos, C.A.; Marques, A.T. Health monitoring of composite structures by embedded FBG and interferometric Fabry-Perot sensors. Comput. Struct. 2008, 86, 340-346. [CrossRef]

8. Posada-Roman, J.; Garcia-Souto, J.A.; Rubio-Serrano, J. Fiber-optic Sensor for Acoustic Detection of Partial Discharges in Oil-Paper Insulated Electrical Systems. Sensors 2012, 12, 4793-4801. [CrossRef] [PubMed]

9. Garcia-Souto, J.A.; Posada, J.E.; Serrano, J.R. All-fiber intrinsic sensor of partial discharge acoustic emission with electronic resonance at $150 \mathrm{kHz}$. Proc. SPIE Opt. Sens. Detect. 2010, 7226, 1-7. [CrossRef]

10. Wei, P.; Han, X.L.; Xia, D. A Measurement Method for the Velocity of Acoustic Emission Wave in Liquid Nitrogen. IEEE Trans. Ind. Electron. 2018, 65, 8232-8238. [CrossRef]

11. Wei, P.; Han, X.L.; Xia, D.; Liu, T.L.; Lang, H. Novel Fiber-Optic Ring Acoustic Emission Sensor. Sensors 2018, 18, 215. [CrossRef]

12. Chen, R.; Theobald, P.; Gower, M. A novel fibre optic acoustic emission sensor. In Proceedings of the SPIE, San Diego, CA, USA, 10-12 March 2008; pp. 1-12.

13. Fu, T.; Liu, Y.J.; Lau, K.T.; Leng, J.S. Impact source identification in a carbon fiber reinforced polymer plate by using embedded fiber optic acoustic emission sensors. Compos. Part B 2014, 66, 420-429. [CrossRef]

14. Yu, F.; Okabe, Y. Fiber-Optic Sensor-Based Remote Acoustic Emission Measurement in a $1000{ }^{\circ} \mathrm{C}$ Environment. Sensors 2017, 17, 2908. [CrossRef]

15. Li, W.; Xu, C.; Ho, S.C.M.; Wang, B.; Song, G. Monitoring Concrete Deterioration Due to Reinforcement Corrosion by Integrating Acoustic Emission and FBG Strain Measurements. Sensors 2017, 17, 657. [CrossRef] [PubMed]

16. Wu, Q.; Okabe, Y. High-sensitivity ultrasonic phase-shifted fiber Bragg grating balanced sensing system. Opt. Express 2012, 20, 28353-28362. [CrossRef] [PubMed]

17. Tsuda, H.; Kumakura, K.; Ogihara, S. Ultrasonic Sensitivity of Strain-Insensitive Fiber Bragg Grating Sensors and Evaluation of Ultrasound-Induced Strain. Sensors 2010, 10, 11248-11258. [CrossRef] [PubMed]

18. Li, Z.X.; Pei, L.; Dong, B.; Ma, C.; Wang, A.B. Analysis of ultrasonic frequency response of surface attached fiber Bragg grating. Appl. Opt. 2012, 51, 4709-4714. [CrossRef] [PubMed]

19. Perez, I.M.; Cui, H.L.; Udd, E. Acoustic emission detection using fiber Bragg gratings. In Proceedings of the SPIE-Smart Structures and Materials, Newport Beach, CA, USA, 6 August 2001; Volume 4328, pp. 209-235.

20. Duff, A.L.; Hamdi, S.E.; Plantier, G.; Lascoup, B. Time delay estimation for acoustic source location by means of short-time cross-correlation. In Proceedings of the IEEE Sensors, Limerick, Ireland, 28-31 October 2011; pp. 1885-1888.

21. Yang, D.; Wang, S.; Lu, P.; Liu, D.M. Dual fiber Bragg gratings configuration-based fiber acoustic sensor for low-frequency signal detection. In Proceedings of the SPIE/COS Photonics Asia, Beijing, China, 9-11 October 2014; Volume 9274, pp. 1-8.

22. Zhao, Y. Optical Fiber Grating and Sensing Technology, 1st ed.; National Defense Industry Press: Beijing, China, 2007; pp. 23-26, ISBN 7-118-04871-2.

23. Wu, Q.; Okabe, Y.; Saito, K.; Yu, F. Sensitivity distribution properties of a phase-shifted fiber bragg grating sensor to ultrasonic waves. Sensors 2014, 14, 1094-1105. [CrossRef] [PubMed]

24. Rosenthal, A.; Razansky, D.; Ntziachristos, V. High-sensitivity compact ultrasonic detector based on a pi-phase-shifted fiber Bragg grating. Opt. Lett. 2011, 36, 1833-1835. [CrossRef] [PubMed]

25. Falcetelli, F.; Romero, M.B.; Pant, S.; Troiani, E.; Martinez, M. Modelling of Pencil-Lead Break Acoustic Emission Sources using the Time Reversal Technique. In Proceedings of the 9th European Workshop on Structural Health Monitoring, Manchester, UK, 10-13 July 2018; pp. 1-13.

26. Innes, M.; Davisa, C.; Rosalie, C.; Norman, P.; Rajic, N. Acoustic Emission Detection and Characterisation Using Networked FBG Sensors. Procedia Eng. 2017, 188, 440-447. [CrossRef]

27. Yang, M.W. Acoustic Emission Detection, 1st ed.; China Machine Press: Beijing, China, 2010; pp. 18-21, ISBN 978-7-111-15772-4.

(C) 2018 by the authors. Licensee MDPI, Basel, Switzerland. This article is an open access article distributed under the terms and conditions of the Creative Commons Attribution (CC BY) license (http://creativecommons.org/licenses/by/4.0/). 